\title{
BATCH NORMALIZED CONVOLUTION NEURAL NETWORK FOR LIVER SEGMENTATION
}

\author{
Fatima Abdalbagi ${ }^{1}$, Serestina Viriri ${ }^{2}$ and Mohammed Tajalsir Mohammed ${ }^{3}$ \\ ${ }^{1}$ Department of Computer Science, Sudan University of Science \\ and Technology, Khartoum, Sudan \\ ${ }^{2}$ Computer Science Discipline, University of KwaZulu-Natal, Durban, South Africa \\ ${ }^{3}$ Department of Computer Science, Sudan University of Science \\ and Technology, Khartoum, Sudan
}

\begin{abstract}
With the huge innovative improvement in all lifestyles, it has been important to build up the clinical fields, remembering the finding for which treatment is done; where the fruitful treatment relies upon the preoperative. Models for the preoperative, for example, planning to understand the complex internal structure of the liver and precisely localize the liver surface and its tumors; there are various algorithms proposed to do the automatic liver segmentation. In this paper, we propose a Batch Normalization After All - Convolutional Neural Network (BATA-Convnet) model to segment the liver CT images using Deep Learning Technique. The proposed liver segmentation model consists of four main steps: pre-processing, training the BATA-Convnet, liver segmentation, and the postprocessing step to maximize the result efficiency. Medical Image Computing and Computer Assisted Intervention (MICCAI) dataset and 3DImage Reconstruction for Comparison of Algorithm Database (3D-IRCAD) were used in the experimentation and the average results using MICCAI are $0.91 \%$ for Dice, $13.44 \%$ for VOE, $0.23 \%$ for RVD, $0.29 \mathrm{~mm}$ for ASD, $1.35 \mathrm{~mm}$ for RMSSD and $0.36 \mathrm{~mm}$ for MaxASD. The average results using $3 D$ IRCAD dataset are $0.84 \%$ for Dice, $13.24 \%$ for VOE, $0.16 \%$ for RVD, $0.32 \mathrm{~mm}$ for ASD, $1.17 \mathrm{~mm}$ for RMSSD and $0.33 \mathrm{~mm}$ for MaxASD.
\end{abstract}

\section{KEYWORDS}

Liver Segmentation, Convolutional Neural Network, Computed Tomography

\section{INTRODUCTION}

Usually, the liver segmentation process done manually by trained clinicians but it is time consuming and requiring much effort and also it different from one clinician to another because of the observer variability; as the result of that, an automatic liver segmentation system would thus be a great boon for perform these tasks. Because of complexity of liver shapes and variable liver sizes among patients the segmentation of the liver from medical images is very difficult and also due to low contrast between the liver and surrounding organs like stomach, pancreas, kidney and muscles. The liver is one of the biggest and an essential organ in the human body. It is molded like a cone and situated in the upper right-hand part of the stomach cavity, underneath the stomach. It is responsible for carrying out some very important functions to keep the body pure of toxins and harmful substances [1]. In excess of 500 real capacities have been related to the liver and some of them remember guideline of most compound levels for the blood, discharge of bile and separating blood that originates from the stomach related parcel [2]. Modern surgeries rely on Computer Aided Diagnosis (CAD) systems to assist doctors in the diagnosis of medical images and surgical planning. CAD it is one of the major research topics because it is part of the routine 
Signal \& Image Processing: An International Journal (SIPIJ) Vol.11, No.5, October 2020

clinical work in medical imaging and diagnostic radiology [3]. Liver pathologies such as cirrhosis, liver cancer and fulminant hepatic failure can be diagnosed by using non-invasive techniques (Medical imaging) such as computed tomography (CT), Magnetic Resonance Imaging (MRI), Ultrasound (US), Positron Emission Tomography (PET) or Single-Photon Emission Computed Tomography (SPECT). One of the advantages of CT images is their higher signal to noise ratio, better spatial resolution and they provide more accurate anatomical information about the visualized structures; therefor, the diagnosticians are preferred to use a CT image [4].

Accurate, efficient and automatic methods for liver segmentation are demanded because traditionally the radiologists and physicians have to manually delineate the liver region slice by slice, which is tedious and time consuming due to the large amount of data [5]. There are several clinical applications for automatic liver segmentation such as, measuring the graft volume before living donor liver transplantations [6], locating vessels and tumors, it is also useful to monitor patients with liver metastases, where the disease is related to an enlargement of the liver, and it plays an important role in surgery planning which it is most time consuming step [7]. Information about precise size for liver, localize the tumors and accurate liver surface segmentation are required in liver local treatment [8]. Due to unpredictability of liver shapes and variable liver sizes among patients the segmentation of the liver from medical images is troublesome because of low differentiation between the liver and encompassing organs like stomach, pancreas, kidney and muscles [9]. Moreover, challenge is presence large tumors and other liver pathologies because the livers with pathologies are different from healthy ones and that results either on segmentation or over segmentation. In the last few decades, a large variety of semiautomatic and fully automatic approaches have been proposed to improve the liver segmentation procedure, such as: region growing, clustering, deformable models or level sets, statistical shape models (SSMs), probabilistic atlases, graph cuts and recently, deep learning techniques [5] [10].

As of late, deep-learning strategies have been utilized in the field of medical image processing [8]. There are three principles building blocks of deep learning methods. These are Convolutional Neural Network (convnet), Auto Encoder (AE) and Deep Belief Network (DBN). These deep learning architectures as of now have been applied in numerous fields like bio-informatics, speech, audio recognition and computer vision [9]. In this paper we focus on using deep learning techniques to detect and segment the liver and compare the result of proposed model with some of the literature.

The ConvNet has gained significant attention for solving computer vision tasks such as object recognition, classification and segmentation [11] [12]. The proposed method is considered as a binary labelling problem which is assigning the labels to each voxel in original image into either belonging to liver or not, and it will be carried out by using convolutional neural network; resulting on a probability map which identifies the liver surface. The contribution made by this paper is the use of a deep learning technique to segment the liver with new concept which adding a BatchNormalization layer after each convolution layer.

The rest of this paper is organized as follows: the literature review and related work are provided in section 2 and section 3 briefly describes the methods and techniques used in this work. The results are detailed and discussed in section 4. Finally, in section 6 the conclusions are drawn.

\section{LiteratURE REVIEW AND RELATED WORK}

There are many semiautomatic and completely automatic approaches have been proposed to improve the liver segmentation methodology. A new method was proposed in [10], for automatic segmentation of liver using supervoxel based graph cuts. Further in G. Litjens et al. (2017) 
Signal \& Image Processing: An International Journal (SIPIJ) Vol.11, No.5, October 2020

mentioned that the new method automatically extracts the Liver Volume of Interest (VOI) and the foreground/background seed points for graph cuts. Firstly, they determined the region of abdomen by using the Maximum Intensity Projection (MIP) and thresholding methods. And extract the specific liver VOI from the region of abdomen according to prior knowledge about locating liver organ and by using a histogram based adaptive thresholding method and morphological operations. They generated the supervoxels of the liver VOI by using the Simple Linear Iterative Clustering (SLIC) method. Besides, foreground/background seeds for graph cuts were chosen on the largest liver slice, and the graph cuts algorithm was applied to the VOI supervoxels.

A Fully automatic CT liver segmentation using a novel statistical shape model approach was presented by [13], they combine learned local shape constraints with observed shape deviation during adaptation. This study [14] introduced a CNN for liver segmentation on abdominal computed tomography (CT) images with focusing on the performance of generalization. They show high generalization of performance and accuracy. They proposed an auto-context neural network; it achieved an effective estimation to obtain the shape prior. They use a self-supervised contour scheme to extend their network. They achieved better accuracy when compared to the state-of-the-art networks by reducing $10.31 \%$ of the Hausdorff distance.

A novel 3D deeply supervised network (DSN) for automatic liver segmentation was presented by [15]. This method employs a fully convolutional architecture to produce a high-quality score map which is processed further by the employment of a conditional random field (CRF) to obtain a refined segmentation. The MICCAI-SLiver07 dataset was used. This method was trained on twenty CT scans and test on ten CT scans. Although the 3D DSN generates high-quality probability maps, a graphical model is employed to re ne segmentation results due to imprecise segmentation in ambiguous regions. A fully connected CRF model on the transverse plane is exploited which solves energy function to re ne the segmentation. The segmentation results achieved were a VOE of $5.42 \%$ and an average symmetric surface distance of $0.79 \mathrm{~mm}$.

The authors in [16] proposed a new 3D liver segmentation method based on multi-region appearance and graph cuts approach in order to reducing user interaction and improving the accuracy and efficiency. The liver could contain tumor or metastasis which results liver with multiple sub-regions, for such case they introduced a novel multiregion-appearance model and appearance selection scheme to segmenting the target multiregion object. The graph cuts approach was used to optimize the proposed energy function. They had compared their work with other graph cuts based methods, state-of-the-art semiautomatic and interactive methods and with prior model based methods. They found that the proposed model needs only initial seeds in the liver when compared with the other graph cuts methods and it required low interactive compared with the other semiautomatic and interactive methods and also the proposed model can be applied to livers with any shape because it was not restricted by specific training data when compared with prior model based methods.

In [17], a fully automatic framework was proposed for liver segmentation based on 3D convolutional neural network (CNN) and globally optimized surface evolution. Firstly, the deep 3D CNN gives the initial liver surface after it was trained to learn a subject-specific probability map of the liver. Then, refining the initial liver segmentation by using the prior information about novel energy function; Finally, propagated the initial liver surface to the optimal position by minimized the energy function using a global optimization-based approach. A semi-automatic method to identify tumors from 3D CT scans based on 2D region growing with knowledge-based constraints was proposed by [18]. Firstly, they decompose the 3D scan image into component slices. Then, they apply 2D region growing with knowledge-based constraints on each slice. 
Signal \& Image Processing: An International Journal (SIPIJ) Vol.11, No.5, October 2020

Finally, they load up the individual segmented lesions together to generate a 3D volume. The region-growing approach used to calculate the seed point and feature vectors and also to label the voxels. The Knowledge-based constraints were used to ensure that the segmented region size and shape is within acceptable parameters. The method required a minimal user involvement in order to define an approximate region of interest around the lesion in each slice image which improved the performance of region growing, as well as reduces computational requirements.

An application based of minimal surfaces and Markov random fields to segmentation the liver tumors were offered in [19]. They were using a region graph instead of a pixel graph in applying these models which leads to an interactive method used to segmenting tumors in 3D CT. their procedure was rely upon the manual definition of a sub-volume containing one or more tumors that need to be segmented based on simple observation which is: the liver presents two types of tissues: tumoral and healthy tissues to classification the liver pixels they were model the liver pixels as a Markov Random Field and the classification is performed through the maximum a posteriori estimation. But this classification step is supervised by user in defining the markers that specify both tumoral and healthy tissues. These markers are used to locate the tumors and to estimate the grey levels characteristics of these structures. They compared their work with the radiologist's segmentations on a set of $5 \mathrm{CT}$ images presenting 10 tumors and found that the mean surface distance between their segmentations and the radiologists is approximately $1.5 \mathrm{~mm}$. approximately $71 \%$ of their segmentations is in perfect match with the radiologist's segmentation. The total mean score obtained on the training data set was equal to 88 . And the computation time needed for the tumor segmentation is approximately equal to five, up to height minutes.

Christ et al. ware presented a combined automatic segmentation of the liver and its lesions in CT and MRI abdomen images using two cascaded fully convolutional neural networks (CFCNs) one for the segmentation of the liver and the other for its lesions [20]. They use an abdominal CT dataset comprising 100 hepatic tumor volumes for training the CFCN models. In the first step of their work they preprocessed the CT/MRI image with HU-windowing or N4 bias correction. Then in second step, segmenting the liver from abdomen CT/MRI scans using first pertained FCN after that they use the output of last step to be input for a second FCN which segments lesions from the given segmented liver ROI. And finally last step was the post-processing using 3D Conditional Random Field (3D CRF). Their results show that the CFCN achieves Dice scores over $94 \%$ for the liver with computation times below 100s per volume. And in contrast to prior work their method could be generalized to segment multiple organs in medical data using multiple cascaded FCNs.

A literature review of medical image segmentation based on U-net was presented by [21]. They were focused on the successful segmentation experience of U-net in six medical imaging systems including computed tomography (CT), magnetic resonance imaging (MRI), ultrasound, X-ray, optical coherence tomography (OCT), and positron emission computed tomography (PET). There are many kinds of lesion regions extracted by these applications. Also in this study, they were introduced the method of combining the original U-net architecture with deep learning and a method for improving the U-net network. They came out that the six imaging systems mentioned in their article, are not perfect in some imaging systems. Consequently, it needs to be improved in future studies for application in various imaging systems.

Two deep encoder-decoder convolutional neural networks (EDCNN) were constructed and trained to cascade segments of both the liver and lesions in CT images in this paper [22]. The first EDCNN was responsible for segmented the liver image which has been an input for the training of a second EDCNN; where it then responsible for segments the tumor regions within the liver 
Signal \& Image Processing: An International Journal (SIPIJ) Vol.11, No.5, October 2020

ROI regions as predicted by the first EDCNN. In order to evaluate their performance, the proposed EDCNN networks produced an average DICE score of $95.22 \%$ for the test set of CT images using a public dataset (3DIRCADb) and it was compared with some of the existing methods. The experimental results demonstrated that the proposed EDCNN achieved improved performance in segmentation accuracy over some existing methods.

The problem of liver segmentation is extremely difficult thus there is no one good solution till now. There are many whose have presented some of the most popular techniques which attempt to deal with this problem like in [35] although they produce reasonable segmentations in many situations but at some point local ambiguities and errors introduced by the segmentation process. And more work needs to be done to match the human detection performance [36]. Still some methods have under-segmentation or over-segmentation errors [37]. These errors need to be reduced in order to improve the accuracy of the segmentation process and that what we try to do in this paper.

The liver segmentation is very complicated so we had to make use of some of the new techniques presented here in this paper. We propose a model based on deep learning technique. Recently, deep learning has achieved state-of-the-art performance in medical image analysis and have been applied to medical image analysis with the intention of letting computers learn the features that optimally represent the problem data at hand [38].

\section{MeThODS AND TECHNIQUES}

Liver segmentation model is implemented in this paper using Batch Normalization Convolutional Neural Network (BATA-Convnet). Our Methodology for Liver segmentation consists of four steps as shown in the flowchart in Figure 1. First, pre-processing the CT image then, training the convnet Architecture. Third step is the liver segmentation. Last step is post processing step to maximize the result efficiency.

\subsection{Datasets}

The datasets used in this work are the Medical Image Computing and Computer Assisted Intervention (MICCAI) 2007 grand challenge dataset and 3D Image Reconstruction for Comparison of Algorithm Database (3D-IRCAD). All CT images in MICCAI are enhanced with different operator and scanned in the focal venous stage on an assortment of scanners (different makers, 4, 16 and 64 detector lines). As it is CT, all datasets were acquired in cross-over direction. The pixel dispersing varied somewhere in the range of 0.55 and $0.80 \mathrm{~mm}$ and interslice distance varied from 1 to $3 \mathrm{~mm}$. The majority of the images is pathologic and includes tumors, metastasis and sores of different sizes. The dataset used for preparing is the MICCAI 2007 grand challenge preparing set (MICCAI-Training) which comprises of 20 volume images with corresponding ground truth segmentations. The dataset used for testing is the MICCAI 2007 grand challenge testing data (MICCAI-Testing) which comprises of 10 volume images. The 3DIRCAD database is composed of the CT scan of 20 patients (10 men and 10 women) with hepatic tumours in $75 \%$ of cases. The images are provided by the authors in DICOM and VTK format in $512 \times 512$ pixels. It also contains handmade true segmentation for liver, bones, tumors and others by medical specialists for all images of the 20 patients. The CT image in 3D-IRCAD is $512 \times 512$, but may take a very long time to complete training such as images on personal computer, so the images were scaled down to $128 \times 128$ pixels. 
Signal \& Image Processing: An International Journal (SIPIJ) Vol.11, No.5, October 2020
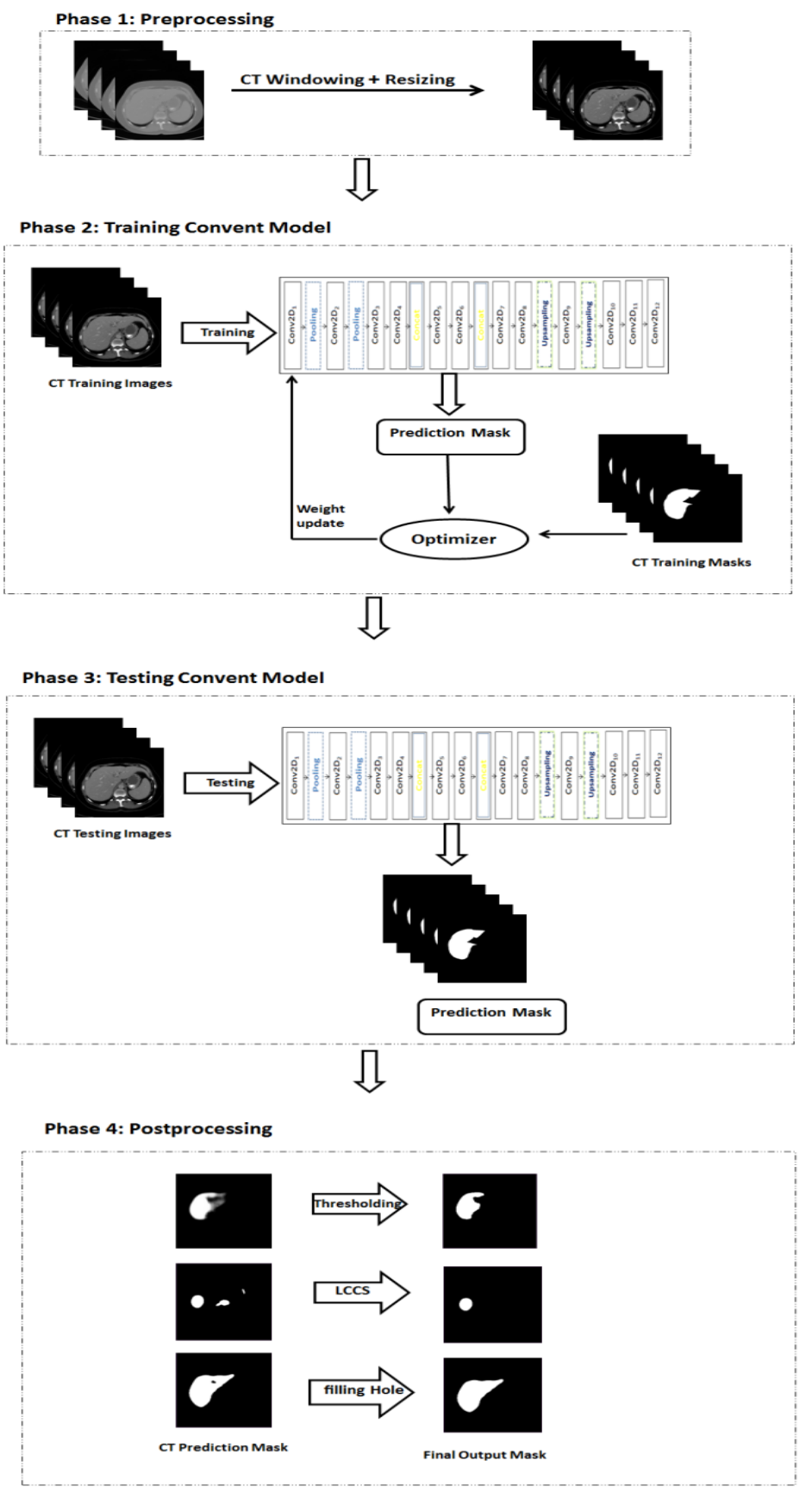

Figure.1 The flowchart of proposed model.

\subsection{Pre-Processing}

In spite of the fact that convnet require significantly less image preprocessing than customary techniques, this is as yet a fundamental errand that can improve results. The CT volumes included the crude information of every clinical focus, and thusly, may contain commotion. To filter such unfortunate data, CT Windowing method was utilized.

Windowing is also known as contrast stretching, grey-level mapping, histogram modification or contrast enhancement is a significant methodology in a CT scan which is the process in which the 
Signal \& Image Processing: An International Journal (SIPIJ) Vol.11, No.5, October 2020

CT image Grayscale component of an image is manipulated via the CT numbers, Pixel intensity measured in Hounsfield Units (HU). Depending on many experimental Sahi et al [24] propose to use the range $[-62,238] \mathrm{HU}$ and P. F. Christ et al. was truncated the image intensity of all CT scans to the range $[-100,400] \mathrm{HU}[25]$. As such, our CT scans range was set to the range [-150, 230] HU as shown in Figure 2.
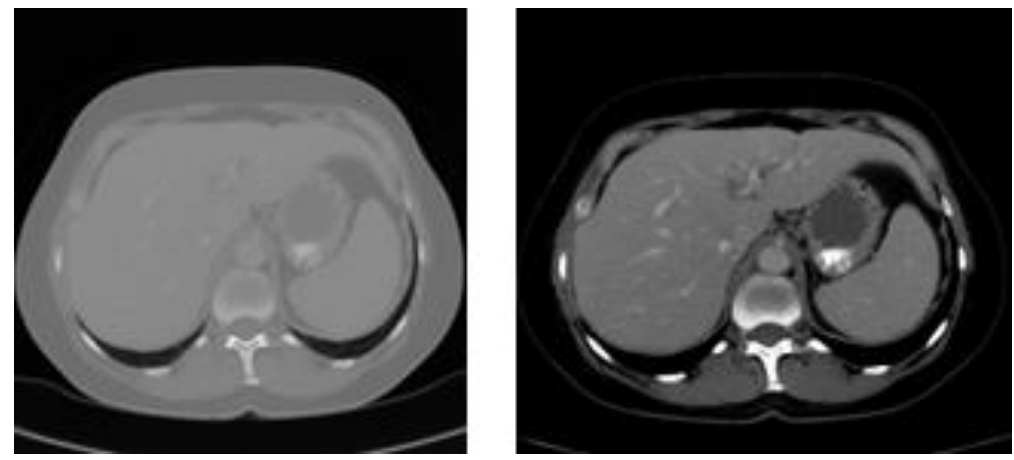

Figure.2 CT Windowing preprocessing

\subsection{Liver Segmentation}

The architecture of the proposed BATA-Convnet as shown in Figure.3 which it includes twelve conv layers (convolution). All convolution layers are followed by a Batch Normalization layer also it's followed by a rectified linear unit (ReLU) to speed up the training. Batch Normalization layer is used to normalizes the values going into each activation function by applying a transformation that maintains the mean output close to zero and the output standard deviation close to one [39]. The network takes an input image of size $128 \times 128$ which corresponds to feature maps of a $2 \mathrm{D}$ slice of the $\mathrm{CT}$ volume.

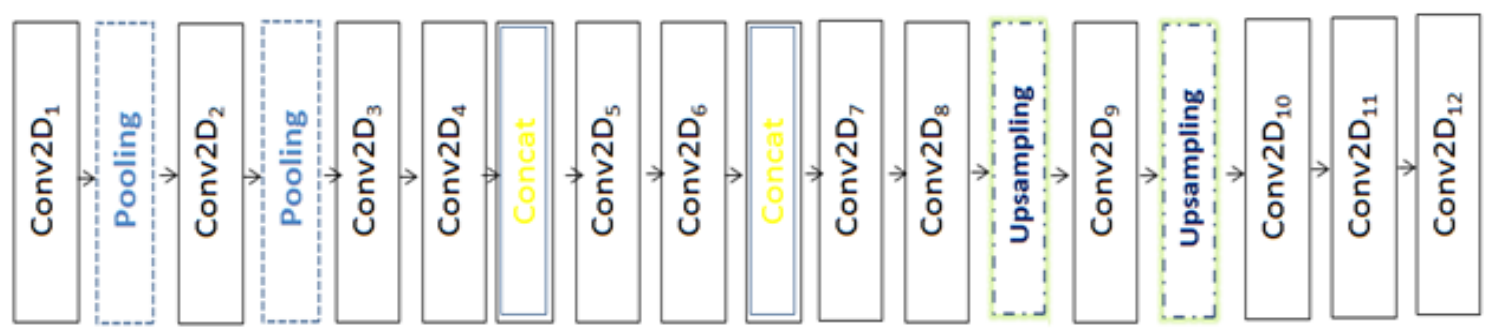

Figure.3 The Architecture of Proposed model.

\subsection{Post Processing}

Post-processing is used to refine the segmentation results produced by the previous segmentation step, including thresholding, largest connected component selection (LCCS) and morphological operations to filling the hole.

\subsubsection{Thresholding}

The Binary Thresholding is used to eliminate the pixels with a probability below 50 percent in the probability map, which in our case is a pixel value 127 which is $50 \%$ of the maximum pixel value of 255 to do that we use an cv2.threshold() function in OpenCV library. The result of the 
thresholding post processing can be seen in Figure 4 where 4(a) and 4(b) are slices before and after thresholding, respectively.

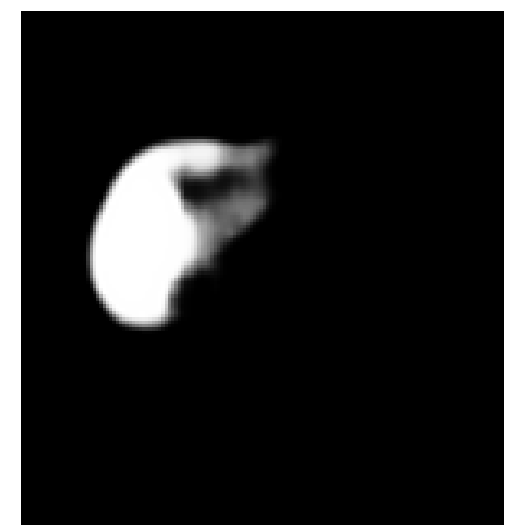

(a)

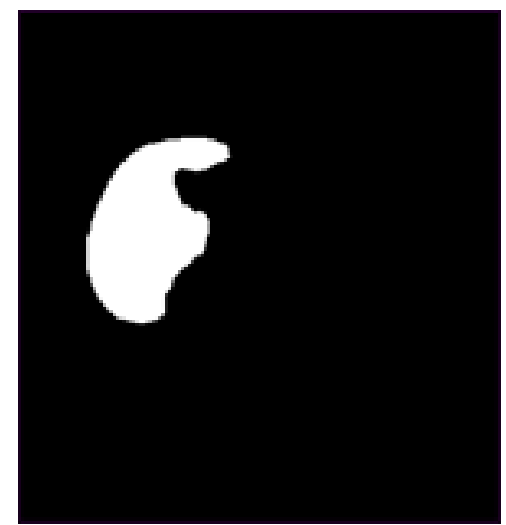

(b)

Figure.4 The Result of the thresholding.

\subsubsection{Largest Connected Component Selection (Lccs)}

The Largest connected component selection (LCCS) technique has been used as a primary segmentation or as post-processing. In our case we used it as post-processing to refine the final result. We calculate the Largest connected component in resulting segmentation volume by setting the background pixel intensity to zero and each component that is not zero within the volume is labeled. Then using python Image Processing Toolbox to calculate the volume of each component and the component with the largest volume is selected. This volume is then set as the threshold volume and all components with a volume less than this threshold volume are removed using morphological operators, this is done using remove_small_objects () function in the image processing Python package scikit-image. The result of the LCCS can be seen in Figure. 5 where 5(a) and 5(b) are slices before and after LCCS process, respectively.

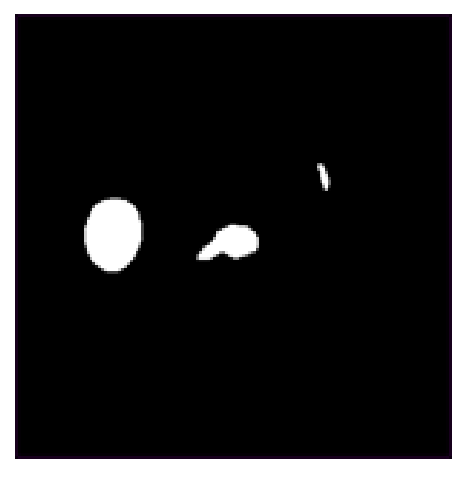

(a)

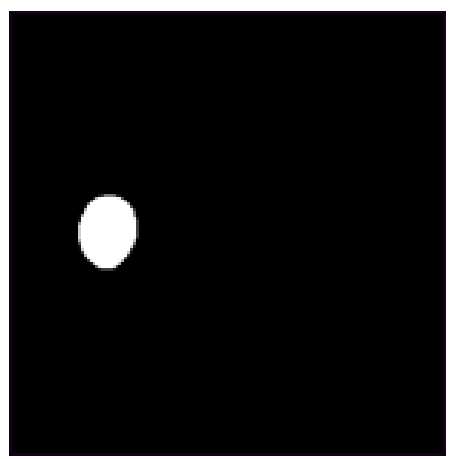

(b)

Figure.5 The result of the LCCS process.

\subsubsection{Filling the Hole}

After the largest connected component is detected and the smaller components are removed, the resulting volume is processed using Morphological closing to remove the hole from it by using 
Signal \& Image Processing: An International Journal (SIPIJ) Vol.11, No.5, October 2020

remove_small_holes() function in scikit-image. The result of the holes filling process can be seen in Figure 6 where 6(a) and 6(b) are slices before and after the process, respectively.

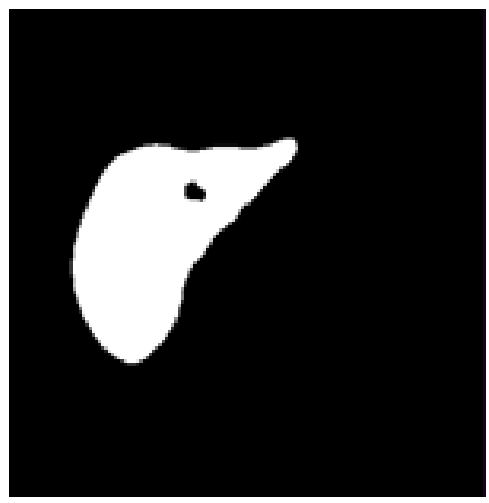

(a)

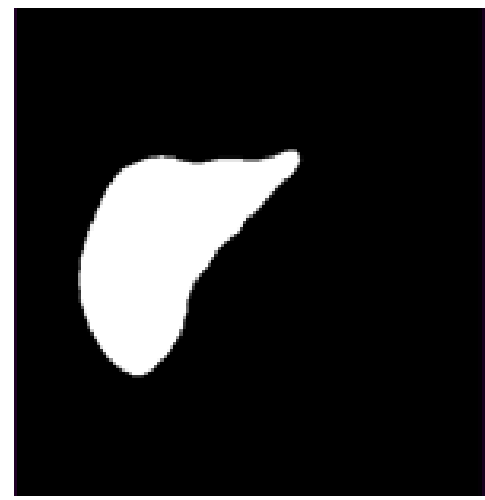

(b)

Figure.6 The result of the Holes Filling process.

\section{RESUlts AND Discussion}

Our proposed BATA-Convnet model for Liver Segmentation consist of twelve conv layers (convolution), two maxpooling layers, two upsampling layers, two concatenate layers as well as a softmax layer. It's implemented in laptop with processor Intel Core(TM) i7-6500U and CPU @ $2.59 \mathrm{GHz}$ with $8.00 \mathrm{~GB}$ RAM and 64 bit operating system. The implementation is done using Python Programming Language and OpenCV library for programming functions with Keras and Tensorflow open-source neural-network library for the implementation of deep learning techniques.

\subsection{Experimental Setup}

An experiment was set up to test BATA-Convnet model for Liver Segmentation in a scientific manner. The model was trained and validated on MICCAI and 3D-IRCAD datasets described above. The network is trained using $75 \%$ of training and the rest for validation. The Training and validation loss and accuracy curves for MICCAI and 3D-IRCAD datasets are depicted in Figure.7 and Figure.8, respectively.
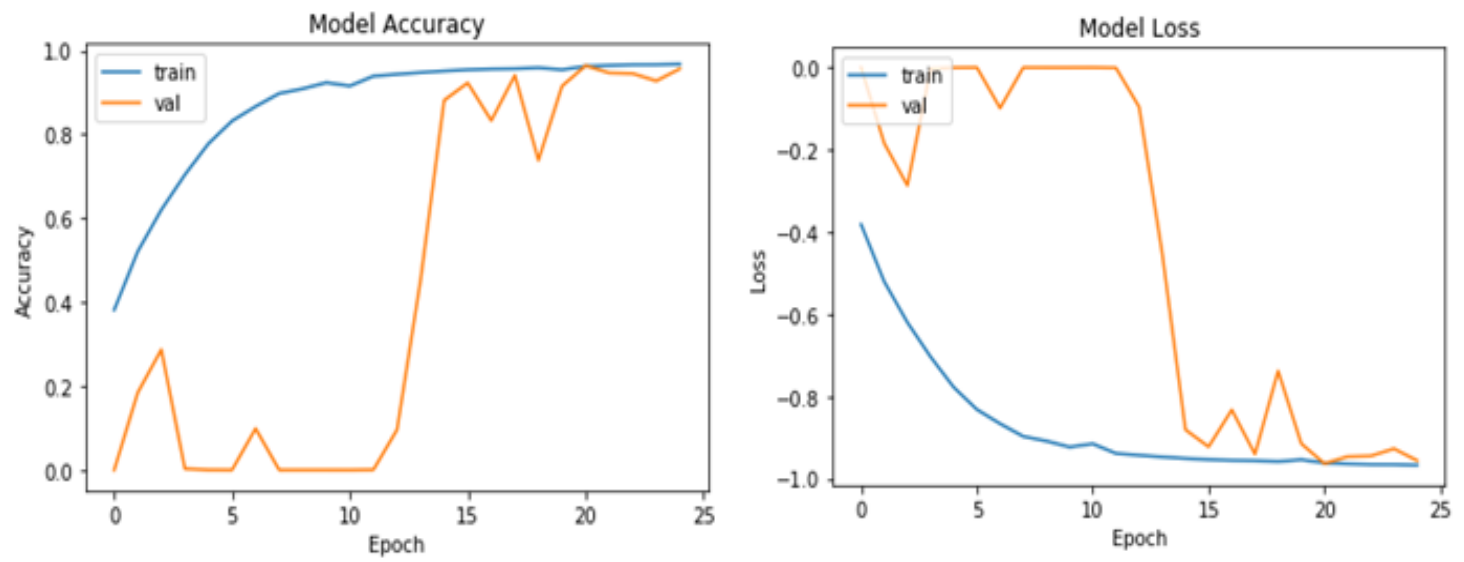

Figure.7 The Training, validation loss and accuracy curves for MICCAI dataset. 
Signal \& Image Processing: An International Journal (SIPIJ) Vol.11, No.5, October 2020
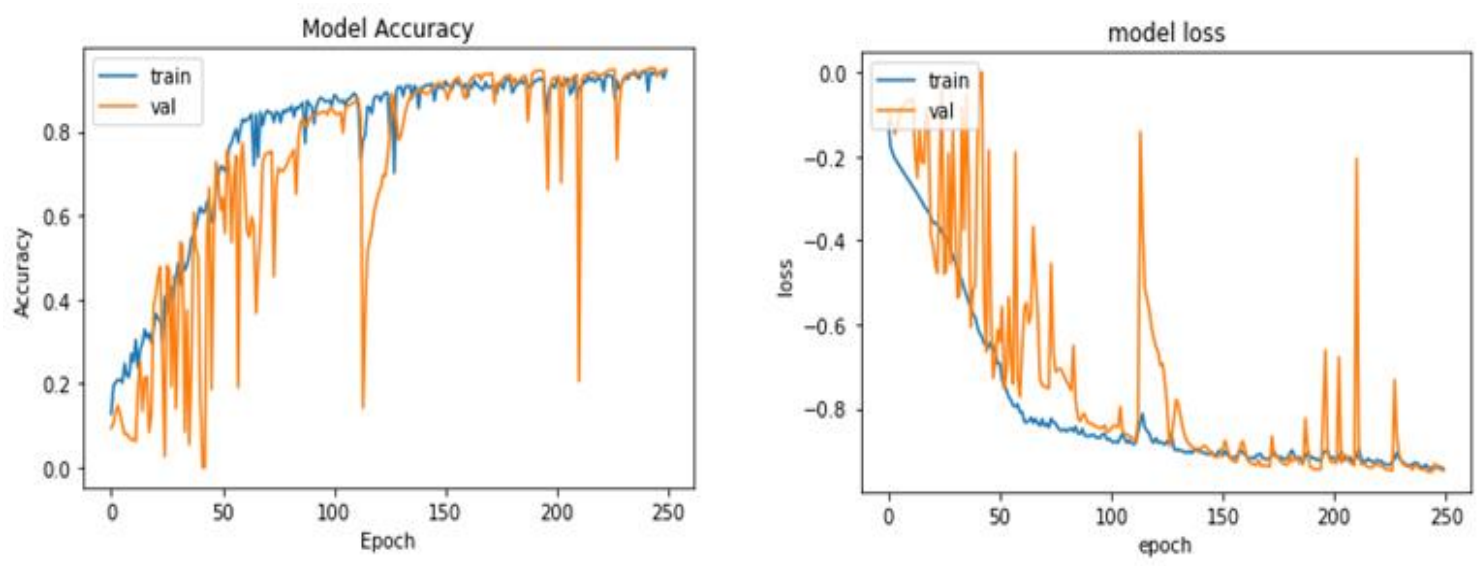

Figure.8 The Training, validation loss and accuracy curves for 3D-IRCAD dataset.

During each iteration $128 \times 128$ slices is randomly chosen from the training data and $128 \times 128$ slices from the ground truth slices as the output. Training the network takes approximately 45 hours using the laptop described above. During the training the validation errors or loss are used to evaluate the network. After training the model, the networks are used to segment the liver in the 2D slices and the probability maps obtained for MICCAI and 3D-IRCAD datasets are depicted in Figure 9 and Figure 10, respectively. Where Figure 9(a), 9(b) and 9(c) are the original images and 9(d), 9(e) and 9(f) are the probability maps obtained by BATA-Convnet model, respectively. And Figure 10(a), 10(b) and 10(c) are the original images and 10(d), 10(e) and 10(f) are the probability maps obtained by BATA-Convnet model, respectively.

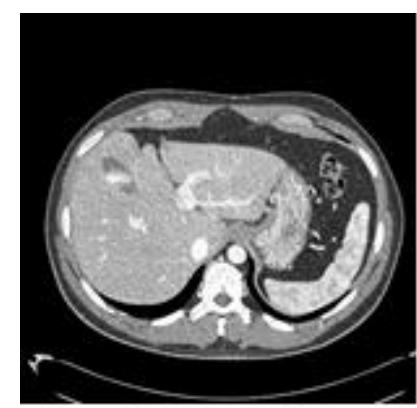

(a)

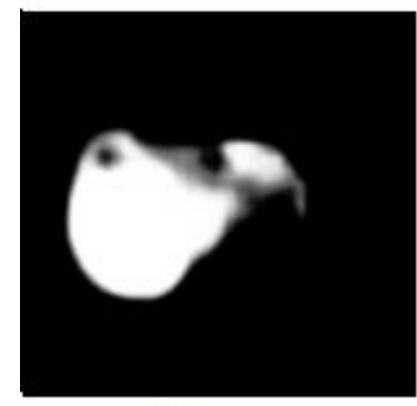

(d)

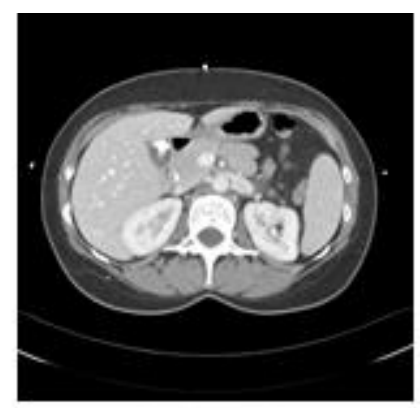

(b)

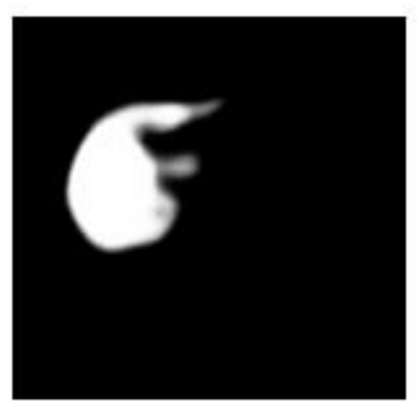

(e)

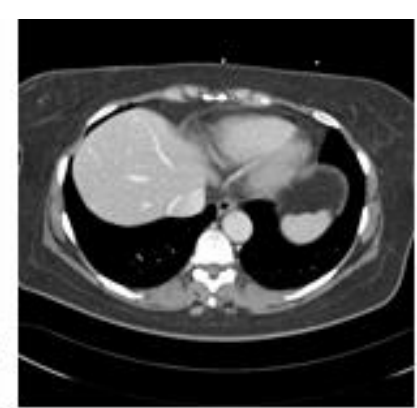

(c)

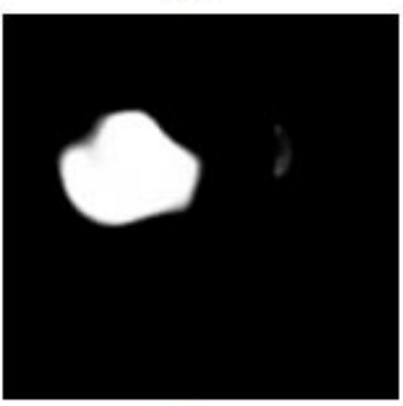

(f)

Figure.9 Sample of probability maps from MICCAI dataset. 
Signal \& Image Processing: An International Journal (SIPIJ) Vol.11, No.5, October 2020

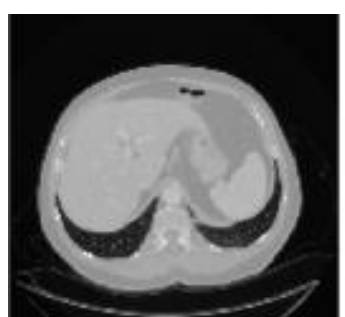

(a)

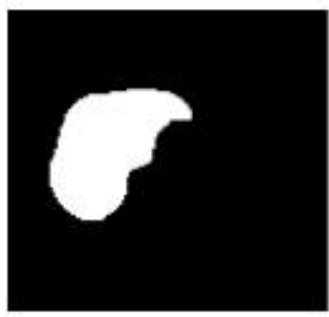

(d)

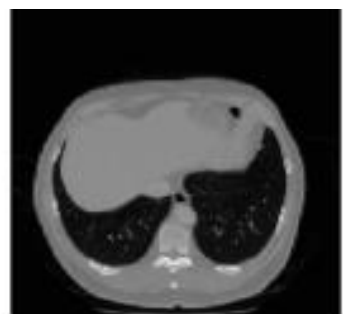

(b)

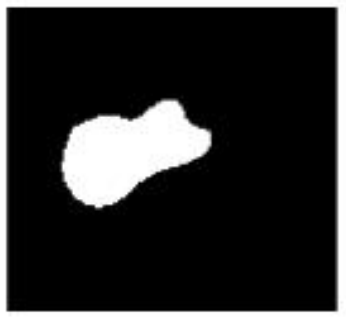

(e)

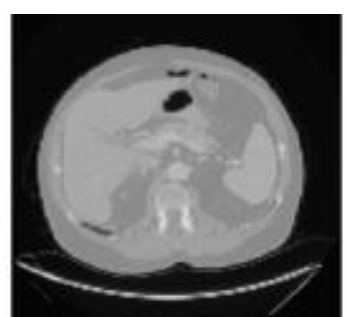

(c)

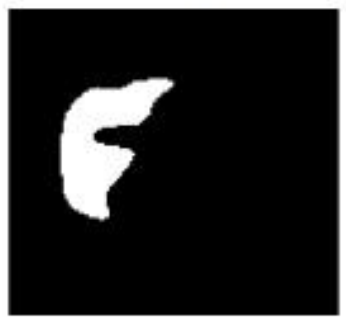

(f)

Figure.10 Sample of probability maps from 3D-IRCAD dataset.

Subsequently, the resulting segmented volumes are compared to the ground truth volumes using some evaluation metrics such as: volume overlap error (VOE), relative volume difference (RVD), maximum symmetric surface distance (Max-ASD), average symmetric surface distance (ASD) and root mean square symmetric surface distance (RMSSD)[26]. The average results using MICCAI are $0.91 \%$ for Dice, $13.44 \%$ for VOE, $0.23 \%$ for RVD, $0.29 \mathrm{~mm}$ for ASD, $1.35 \mathrm{~mm}$ for RMSSD and $0.36 \mathrm{~mm}$ for MaxASD. The average result using 3D-IRCAD dataset are $0.84 \%$ for Dice, $13.24 \%$ for VOE, $0.16 \%$ for RVD, $0.32 \mathrm{~mm}$ for ASD, $1.17 \mathrm{~mm}$ for RMSSD and $0.33 \mathrm{~mm}$ for MaxASD. Thereafter, the result was compared to some related literature [16] [19] [27] [28] [29] [30] [31] [32] [33] [34] as shown in table I. The results in terms of volume overlap error were better than those results [28] [19] [31] as shown in Figure 11 and in terms of the relative volume difference were better than those results [27] [30] [31] as shown in Figure 12. But in in terms of (ASD), (RMSSD) and (Max-ASD) our result is better than all the other results as shown in Figure 13, Figure 14 and Figure 15, respectively

Table 1: Comparison to related literature

\begin{tabular}{|c|c|c|c|c|c|}
\hline Related Work & VOE & RVD & ASD & RMSSD & Max-ASD \\
\hline$[16]$ & 4.53 & 0.15 & 0.79 & 1.49 & 16.75 \\
\hline$[27]$ & 7.87 & 1.31 & 1.29 & 2.50 & 23.56 \\
\hline$[28]$ & 72.38 & - & - & - & - \\
\hline$[19]$ & 29.49 & - & - & - & - \\
\hline$[29]$ & 5.36 & 0.03 & 0.96 & 1.84 & 19.20 \\
\hline$[30]$ & 5.90 & 2.70 & 0.91 & 1.88 & 18.94 \\
\hline$[31]$ & 16.75 & 2.05 & - & - & - \\
\hline$[32]$ & 5.42 & - & 0.79 & - & - \\
\hline$[33]$ & - & - & - & - & - \\
\hline$[34]$ & - & - & - & $\mathbf{0 . 3 6 m m}$ \\
\hline $\begin{array}{c}\text { Result for } \\
\text { MICCAI }\end{array}$ & $\mathbf{1 3 . 4 4 \%}$ & $\mathbf{0 . 2 3 \%}$ & $\mathbf{0 . 2 9 m m}$ & $\mathbf{1 . 3 5 m m}$ & $\mathbf{0 . 3 3 m m}$ \\
\hline $\begin{array}{c}\text { Result for 3D- } \\
\text { IRCAD }\end{array}$ & $\mathbf{1 3 . 2 4 \%}$ & $\mathbf{0 . 1 6 \%}$ & $\mathbf{0 . 3 2 m m}$ & $\mathbf{1 . 1 7 m m}$ & $\mathbf{1 m m}$ \\
\hline
\end{tabular}


Signal \& Image Processing: An International Journal (SIPIJ) Vol.11, No.5, October 2020

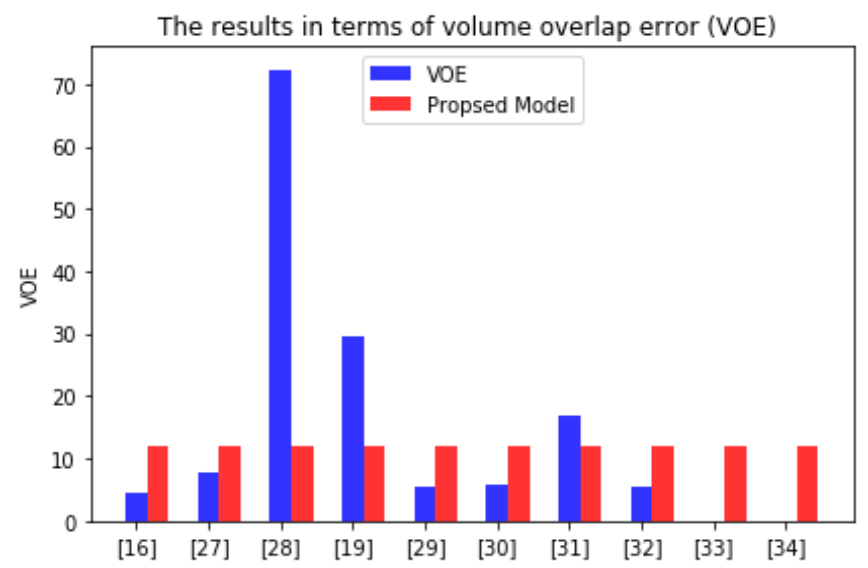

Figure.11 The results in terms of VOE

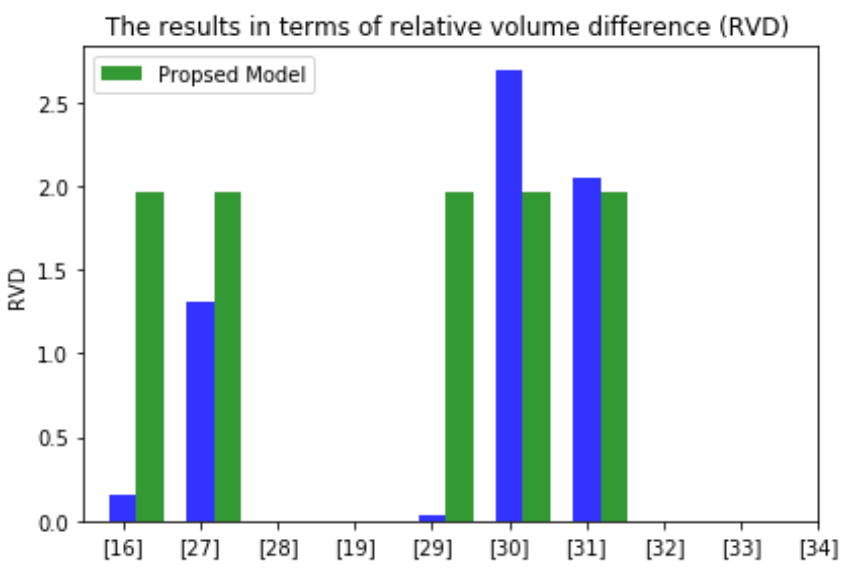

Figure.12 The results in terms of RVD

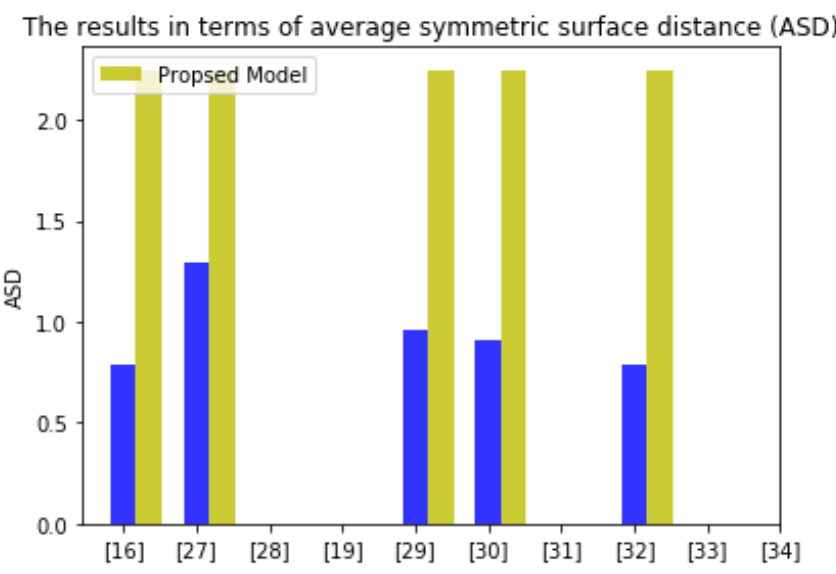

Figure.13 The results in terms of ASD 
Signal \& Image Processing: An International Journal (SIPIJ) Vol.11, No.5, October 2020

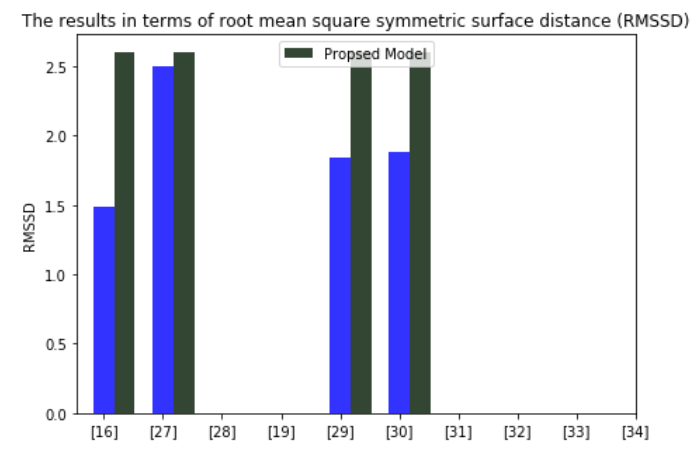

Figure.14 The results in terms of RMSSD

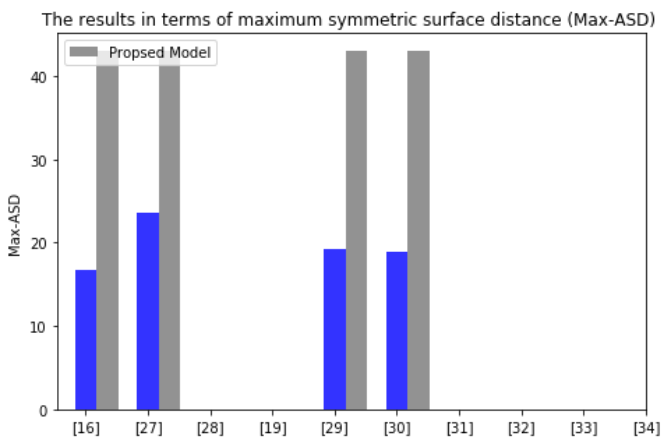

Figure.15 The results in terms of Max-ASD

\section{CONClusion}

The BATA-Convnet model for liver segmentation using 3D CT scans is proposed in this paper. After trying many experiments for the architecture of our network we come out to propose a BATA-Convnet, the ordinary Convnet it's stack of the convolution followed by maxpooling layers here we proposed to add Batch Normalization layer after all convolution layer which enhance the overall accuracy. BATA-Convnet model is implemented using Python Programming Language and OpenCV library for programming functions with Keras and TensorFlow OpenSource Neural-Network library. MICCAI and 3D-IRCAD datasets were used in the experimentation and the average results using MICCAI are $0.91 \%$ for Dice, $13.44 \%$ for VOE, $0.22 \%$ for RVD, $0.29 \mathrm{~mm}$ for ASD, $1.35 \mathrm{~mm}$ for RMSSD and $0.36 \mathrm{~mm}$ for MaxASD. The average result using 3D-IRCAD dataset are $0.84 \%$ for Dice, $13.24 \%$ for VOE, $0.16 \%$ for RVD, $0.32 \mathrm{~mm}$ for ASD, $1.17 \mathrm{~mm}$ for RMSSD and $0.33 \mathrm{~mm}$ for MaxASD.

\section{Conflicts of Interest}

The authors declare no conflict of interest.

\section{REFERENCES}

[1] V. Dixit, J. Pruthi, "Review of Image Processing Techniques for Automatic Detection of Tumor in Human Liver," International Journal of Computer Science and Mobile Computing, 33(3), 371-378, 2014.

[2] Johns Hopkins Medicine, 2019.

[3] K. Doi, "Computer-aided diagnosis in medical imaging: Historical review, current status and future potential," Computerized Medical Imaging and Graphics, 31(4-5), 198-211, 2007, doi:10.1016/j.compmedimag.2007.02.002. 
Signal \& Image Processing: An International Journal (SIPIJ) Vol.11, No.5, October 2020

[4] P. Campadelli, E. Casiraghi, A. Esposito, "Liver segmentation from computed tomography scans: A survey and a new algorithm," Artificial Intelligence in Medicine, 45(2-3), 185-196, 2009, doi:10.1016/j.artmed.2008.07.020.

[5] Y. Nakayama, Q. Li, S. Katsuragawa, et al., "Automated hepatic volumetry for living related liver transplantation at multisection CT.", Radiology, 240(3), 743-748, 2006, doi:10.1148/radiol.2403050850.

[6] L. Hermoye, I. Laamari-Azjal, Z. Cao, L. Annet, J. Lerut, B.M. Dawant, B.E. Van Beers, "Liver Segmentation in Living Liver Transplant Donors: Comparison of Semiautomatic and Manual Methods," Radiology, 234(1), 171-178, 2005, doi:10.1148/radiol.2341031801.

[7] T. Heimann, I. Wolf, H.-P. Meinzer, "Active shape models for a fully automated 3D segmentation of the liver--an evaluation on clinical data.," Proc. Medical Image Computing and Computer-Assisted Intervention, 9(Pt 2), 41-8, 2006, doi:10.1007/11866763_6.

[8] L. Massoptier, S. Casciaro, "A new fully automatic and robust algorithm for fast segmentation of liver tissue and tumors from CT scans," European Radiology, 18(8), 1658-1665, 2008, doi:10.1007/s00330-008-0924-y.

[9] X.Z.X. Zhang, J.T.J. Tian, K.D.K. Deng, Y.W.Y. Wu, X.L.X. Li, A.S. Construction, "Automatic liver segmentation using a statistical shape model with optimal surface detection," Biomedical Engineering, IEEE Transactions on, 57(10), 2622-2626, 2010, doi:10.1109/TBME.2010.2056369.

[10] G. Litjens, T. Kooi, B.E. Bejnordi, A.A.A. Setio, F. Ciompi, M. Ghafoorian, J.A.W.M. van der Laak, B. van Ginneken, C.I. Sánchez, "A Survey on Deep Learning in Medical Image Analysis," (February), 2017, doi:10.1016/j.media.2017.07.005.

[11] J. Long, E. Shelhamer, T. Darrell, "Fully convolutional networks for semantic segmentation," Proceedings of the IEEE Computer Society Conference on Computer Vision and Pattern Recognition, 07-12-June, 3431-3440, 2015, doi:10.1109/CVPR.2015.7298965.

[12] A. Krizhevsky, G.E. Hinton, "ImageNet Classification with Deep Convolutional Neural Networks," $1-9$.

[13] M. Erdt, S. Steger, M. Kirschner, S. Wesarg, "Fast automatic liver segmentation combining learned shape priors with observed shape deviation," Proceedings - IEEE Symposium on Computer-Based Medical Systems, 249-254, 2010, doi:10.1109/CBMS.2010.6042650.

[14] M. Chung, J. Lee, J. Lee, Y.-G. Shin, "Liver Segmentation in Abdominal CT Images via AutoContext Neural Network and Self-Supervised Contour Attention,” 2020.

[15] Q. Dou, H. Chen, Y. Jin, L. Yu, J. Qin, P.A. Heng, “3D deeply supervised network for automatic liver segmentation from CT volumes," Lecture Notes in Computer Science (Including Subseries Lecture Notes in Artificial Intelligence and Lecture Notes in Bioinformatics), 9901 LNCS, 149-157, 2016, doi:10.1007/978-3-319-46723-8_18.

[16] J. Peng, P. Hu, F. Lu, Z. Peng, D. Kong, H. Zhang, “3D liver segmentation using multiple region appearances and graph cuts," Medical Physics, 42(12), 6840-6852, 2015, doi:10.1118/1.4934834.

[17] P. Hu, F. Wu, J. Peng, P. Liang, D. Kong, "Automatic 3D liver segmentation based on deep learning and globally optimized surface evolution," Physics in Medicine and Biology, 61(24), 8676-8698, 2016, doi:10.1088/1361-6560/61/24/8676.

[18] D. Wong, J. Liu, Y. Fengshou, Q. Tian, W. Xiong, J. Zhou, Y. Qi, T. Han, S.K. Venkatesh, S. Wang, "A semi-automated method for liver tumor segmentation based on 2D region growing with knowledge-based constraints," The Midas Journal, (January), 2008.

[19] J. Stawiaski, E. Decencière, F. Bidault, J. Stawiaski, E. Decencière, F. Bidault, I. Liver, T. Segmentation, "Interactive Liver Tumor Segmentation Using Graph-cuts and Watershed To cite this version : HAL Id : hal-01445751 Graph-cuts and Watershed," 2017.

[20] P.F. Christ, F. Ettlinger, F. Grün, M.E.A. Elshaera, J. Lipkova, S. Schlecht, F. Ahmaddy, S. Tatavarty, M. Bickel, P. Bilic, M. Rempfler, F. Hofmann, M.D. Anastasi, S.-A. Ahmadi, G. Kaissis, J. Holch, W. Sommer, R. Braren, V. Heinemann, B. Menze, "Automatic Liver and Tumor Segmentation of CT and MRI Volumes using Cascaded Fully Convolutional Neural Networks," 1$20,2017$.

[21] G. Du, X. Cao, J. Liang, X. Chen, Y. Zhan, "Medical image segmentation based on U-Net: A review," Journal of Imaging Science and Technology, 64(2), 1-12, 2020, doi:10.2352/J.ImagingSci.Technol.2020.64.2.020508. 
Signal \& Image Processing: An International Journal (SIPIJ) Vol.11, No.5, October 2020

[22] Ü. Budak, Y. Guo, E. Tanyildizi, A. Şengür, "Cascaded deep convolutional encoder-decoder neural networks for efficient liver tumor segmentation," Medical Hypotheses, 134(September 2019), 2020 , doi:10.1016/j.mehy.2019.109431.

[23] S. Luo, X. Li, J. Li, "Review on the Methods of Automatic Liver Segmentation from Abdominal Images," Journal of Computer and Communications, 2(2), 1-7, 2014, doi:10.4236/jcc.2014.22001.

[24] K. Sahi, S. Jackson, E. Wiebe, G. Armstrong, S. Winters, R. Moore, G. Low, "The value of 'liver windows' settings in the detection of small renal cell carcinomas on unenhanced computed tomography," Canadian Association of Radiologists Journal, 65(1), 71-76, 2014, doi:10.1016/j.carj.2012.12.005.

[25] P.F. Christ, F. Ettlinger, F. Grün, M.E.A. Elshaera, J. Lipkova, S. Schlecht, F. Ahmaddy, S. Tatavarty, M. Bickel, P. Bilic, M. Rempfler, F. Hofmann, M.D. Anastasi, S.-A. Ahmadi, G. Kaissis, J. Holch, W. Sommer, R. Braren, V. Heinemann, B. Menze, "Automatic Liver and Tumor Segmentation of CT and MRI Volumes using Cascaded Fully Convolutional Neural Networks," (February), 2017.

[26]. M. Styner, J. Lee, B. Chin, M. Chin, “3D segmentation in the clinic: A grand challenge II: MS lesion segmentation," Midas, 1-6, 2008.

[27] W. Wu, Z. Zhou, S. Wu, Y. Zhang, "Automatic Liver Segmentation on Volumetric CT Images Using Supervoxel-Based Graph Cuts," Computational and Mathematical Methods in Medicine, 2016(April), 1-14, 2016, doi:10.1155/2016/9093721.

[28] M. Goryawala, S. Gulec, R. Bhatt, A.J. McGoron, M. Adjouadi, "A Low-Interaction Automatic 3D Liver Segmentation Method Using Computed Tomography for Selective Internal Radiation Therapy," BioMed Research International, 2014(1), 1-12, 2014, doi:10.1155/2014/198015.

[29] P. Hu, F. Wu, J. Peng, P. Liang, D. Kong, "Automatic 3D liver segmentation based on deep learning and globally optimized surface evolution," Physics in Medicine and Biology, 61(24), 8676-8698, 2016, doi:10.1088/1361-6560/61/24/8676.

[30] F. Lu, F. Wu, P. Hu, Z. Peng, D. Kong, "Automatic 3D liver location and segmentation via convolutional neural networks and graph cut," 2016, doi:10.1007/s11548-016-1467-3.

[31] R. Vivanti, A. Ephrat, L. Joskowicz, O. A, J. Sosna, "Automatic liver tumor segmentation in followup CT studies using Convolutional Neural Networks The Rachel and Selim Benin School of Computer Science and Department of Radiology," c, 2015.

[32] Q. Dou, L. Yu, H. Chen, Y. Jin, X. Yang, J. Qin, P. Heng, “3D deeply supervised network for automated segmentation of volumetric medical images," Medical Image Analysis, 41, 40-54, 2017, doi:10.1016/j.media.2017.05.001.

[33] L. Massoptier, S. Casciaro, "Fully automatic liver segmentation through graph-cut technique," Annual International Conference of the IEEE Engineering in Medicine and Biology - Proceedings, 5243-5246, 2007, doi:10.1109/IEMBS.2007.4353524.

[34] L. Massoptier, S. Casciaro, "A new fully automatic and robust algorithm for fast segmentation of liver tissue and tumors from CT scans," 1658-1665, 2008, doi:10.1007/s00330-008-0924-y.

[35] A. Gotra, L. Sivakumaran, G. Chartrand, K.-N. Vu, F. Vandenbroucke-Menu, C. Kauffmann, S. Kadoury, B. Gallix, J.A. de Guise, A. Tang, "Liver segmentation: indications, techniques and future directions,” Insights into Imaging, 8(4), 377-392, 2017, doi:10.1007/s13244-017-0558-1.

[36] Mubashir Ahmad, Yuan Ding, Syed Furqan Qadri, Jian Yang, "Convolutional-neural-network-based feature extraction for liver segmentation from CT images," Proc. SPIE 11179, Eleventh International Conference on Digital Image Processing (ICDIP 2019), 1117934 (14 August 2019); https://doi.org/10.1117/12.2540175

[37] Weiwei Wu, Zhuhuang Zhou, Shuicai Wu, Yanhua Zhang, "Automatic Liver Segmentation on Volumetric CT Images Using Supervoxel-Based Graph Cuts", Computational and Mathematical Methods in Medicine, vol. 2016, Article ID 9093721, 14 pages, 2016. https://doi.org/10.1155/2016/9093721

[38] G. Litjens, T. Kooi, B.E. Bejnordi, A.A.A. Setio, F. Ciompi, M. Ghafoorian, J.A.W.M. van der Laak, B. van Ginneken, C.I. Sánchez, "A Survey on Deep Learning in Medical Image Analysis," (February), 2017, doi:10.1016/j.media.2017.07.005.

[39] S. Ioffe and C. Szegedy, "Batch normalization: Accelerating deep network training by reducing internal covariate shift," 32nd Int. Conf. Mach. Learn. ICML 2015, vol. 1, pp. 448-456, 2015. 